Revue internationale de l'économie sociale

Recma

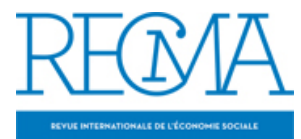

--> Voir l'erratum concernant cet article

\title{
La gouvernance des entreprises coopératives, Sous la direction de Jorge Muñoz, Mario Radrigan Rubio et Yann Regnard. Presses universitaires de Rennes, février 2008
}

\section{Nadine Richez-Battesti}

Numéro 308, mai 2008

URI : https://id.erudit.org/iderudit/1021176ar

DOI : https://doi.org/10.7202/1021176ar

Aller au sommaire du numéro

Éditeur(s)

Association Recma

ISSN

1626-1682 (imprimé)

2261-2599 (numérique)

Découvrir la revue

Citer ce compte rendu

Richez-Battesti, N. (2008). Compte rendu de [La gouvernance des entreprises coopératives, Sous la direction de Jorge Muñoz, Mario Radrigan Rubio et Yann Regnard. Presses universitaires de Rennes, février 2008]. Revue internationale de l'économie sociale, (308), 93-94. https://doi.org/10.7202/1021176ar d'utilisation que vous pouvez consulter en ligne. 
socialement responsable et sa contradiction fondamentale entre profits élevés et mission sociale ne semblent pas non plus à la hauteur du défi à relever, ce qui justifie la nouvelle approche $\mathrm{du}$ " social-business » faisant largement appel aux multinationales et aux grandes fondations anglo-saxonnes en plein développement.

Mais, dans le cadre de l'émergence d'un nouveau discours, et peut-être à terme d'un nouvel " esprit »(9), sur le développement du capitalisme global centré sur l'éthique - le philanthrocapitalisme ${ }^{(10)}$-, les social-business sont censés dépasser non seulement les dérives du capitalisme financier, mais également les formes moins performantes de l'action sociale $-\mathrm{y}$ compris la solidarité internationale-, notamment caritative. Là aussi, on peut analyser la proposition de M. Yunus à l'aune des débats sur la globalisation résumés par M. Abélès (2008). Ses critiques y verront un argument supplémentaire pour légitimer le projet néolibéral prônant l'ouverture et la liberté des échanges et la consolidation du capitalisme sans frontières afin d'élargir son espace géographique. Leurs contradicteurs évoquent le nouvel âge du capitalisme global rythmé par Internet et la mobilité du capital financier dont les principaux acteurs sont les firmes et les banques multinationales.

En ce sens, et dans ses différentes dimensions et ses différentes échelles géographiques - du local au global -, la proposition du social-business et de ses impacts ne saurait se soustraire à l'analyse des gagnants et des perdants qu'elle engendre.

François Doligez

(9) Voir Boltanski L. et Chiapello E., 1999, Le nouvel esprit du capitalisme, Gallimard, Paris, $843 \mathrm{p}$.

(10) Voir l'article de la revue The Economist sur la " naissance du philanthrocapitalisme ", repris dans Problèmes économiques, $n^{\circ} 2$ 912, décembre 2006.

\section{La gouvernance des entreprises coopératives}

Sous la direction de Jorge Muñoz, Mario Radrigan Rubio et Yann Regnard. Presses universitaires de Rennes, février 2008.

Cet ouvrage reprend une sélection de papiers présentés lors d'un colloque à Brest en 2006. Publié aux Presses universitaires de Rennes, qui éditent de façon croissante des ouvrages dédiés à l'économie sociale, il illustre à la fois le regain d'intérêt pour ce mode d'organisation et la richesse des recherches en cours dans le champ des coopératives et plus précisément sur les questions de gouvernance.

Cet ouvrage, à dimension internationale, réunit des contributions de chercheurs de différents pays: France, Espagne, Québec et plus largement Canada, et Chili. Une telle dimension internationale n'est pas anodine, elle exprime l'ancrage coopératif à cette échelle. Plus précisément ici, elle est aussi le fruit d'une dynamique initiée en 2003 concernant la constitution d'un réseau international universitaire des études coopératives (Rulescoop) reliant tout particulièrement l'Europe et l'Amérique centrale et latine. Elle est enfin l'expression d'un questionnement qui dépasse les seules frontières nationales et qui est abordé à la lumière des sciences de gestion, de l'économie ou de la sociologie. Ce questionnement porte sur la capacité des coopératives à se développer sans " perdre leur âme » dans un marché toujours plus concurrentiel et mondialisé et dans un contexte de politiques économiques marquées par une orientation libérale affirmée. Les coopératives sont-elles en capacité d'introduire des formes de régulation, voire de proposer des alternatives productives au modèle dominant, et selon quelles modalités? C'est la question générale qui traverse l'ensemble de l'ouvrage.

Lintérêt de l'ouvrage est aussi de rendre compte de la diversité du mouvement coopératif relativement à ses développements nationaux ou transnationaux, aux différentes formes de coopératives (de producteurs, de consommation, 
d'intercoopération...) ou encore aux secteurs d'activité qu'il concerne. Ainsi sont examinés le secteur de la pêche, celui de l'agroalimentaire plus largement ou encore celui de la banque. Les onze papiers présentés s'articulent autour de deux entrées: la première interroge la gouvernance participative et concerne plus les modes d'organisation internes des coopératives et les modalités d'association de leurs sociétaires; la seconde prolonge l'exploration des liens entre les coopératives et le territoire, thématique d'un ouvrage de 2003 sous la direction de J.-M. Touzard et J.-F. Draperi. On aborde dans ce second volet les relations entretenues avec les parties prenantes externes, qu'elles concernent les pouvoirs publics et la question de la réglementation ou plus largement de la régulation qu'ils sont susceptibles de transformer, des modalités alternatives de développement ou plus généralement l'environnement de l'entreprise coopérative. Le profane y comprendra mieux ce qu' " entreprendre autrement " et solidarité signifient, notamment du point de vue des relations sociales, des influences du sociétariat sur le pilotage de l'organisation et ses spécificités relativement à l'actionnaire, de la relation de long terme qui s'instaure avec l'ensemble des associés et du point de vue de l'ancrage des coopératives dans leur territoire et de leur contribution au développement.
Dans le même temps, pour le lecteur déjà sensibilisé à la question coopérative, les articles illustrent aussi les tensions qui traversent l'ensemble du mouvement coopératif et la diversité des " gammes " en présence au sein du modèle coopératif aujourd'hui. Ils donnent des clés de lecture pour comprendre la diversification des stratégies des organisations coopératives et le caractère polarisé de leur développement sur certains territoires. L'acteur de terrain trouvera de quoi étayer ses interrogations sur les questions de gouvernance et matière à resituer les transformations qu'il observe dans son propre secteur d'activité dans un contexte plus large, en termes de secteur d'activité ou de pays. Enfin, l'universitaire ou le chercheur trouvera matière à nourrir ses propres approches sur la gouvernance et l'entrepreneuriat coopératif.

Au-delà de ces apports immédiats, cet ouvrage ouvre des perspectives quant à l'intérêt des comparaisons internationales, mais aussi concernant celui d'un travail renforcé sur l'évaluation de l'impact sociétal des coopératives, dont on perçoit tout à la fois l'intérêt et les limites des méthodologies et des outils existants. Il souligne plus largement le défi qu'il y a à mieux diffuser la connaissance de ce modèle coopératif, notamment dans les impacts que l'on peut en attendre.

Nadine Richez-Battesti 